\title{
Response to Letter to the Editor: Systemic Adiponectin Values in Humans Require Standardized Units
}

\author{
B. D. M. Netto ${ }^{1}$ - A. R. Dâmaso ${ }^{1,2,3}$
}

Published online: 24 November 2015

(C) Springer Science+Business Media New York 2015

Thank you Dr. Bastard et al. for the insightful comments and concerns about how to express adiponectin values in humans. We have carefully read with great interest your comment, and we would like to thank you for the opportunity to clarify a mistake in our recent research data. In our study, the absolute values of adiponectinemia are correct; however, the unit used to express adiponectin is not appropriate, since the results of adiponectin are expressed in micrograms per milliliter instead of nanograms per milliliter as is described in our article. In addition, we believe to be important to reinforce that many studies published in the literature had also expressed values of adiponectinemia in individuals with extreme obesity in nanograms per milliliter [1-4]. Based on the findings in the literature, we certainly agree that is highly necessary to standardize units to express circulating adiponectin values, thus far this will

B. D. M. Netto

barbaradmnetto@gmail.com

1 Post-Graduate Program of Nutrition, Escola Paulista de Medicina UNIFESP/EPM, Rua Marselhesa, 630 - Vila Clementino, 04020-060 São Paulo, SP, Brazil

2 Biosciences Department, UNIFESP/EPM, São Paulo, Brazil

3 Post-Graduate Program of Interdisciplinary Health Sciences, UNIFESP/EPM, São Paulo, Brazil

provide more robust comparisons and consistency among studies.

Best regards,

Netto, BDM, Msc.; Dâmaso, A.R., Post-Ph.D

\section{Compliance Ethical Standards}

Conflict of Interest The authors declare that they have no competing interests.

Ethical Approval For this type of paper, formal consent is not required.

\section{References}

1. Hosseinzadeh-Attar MJ, Golpaie A, Janani L, et al. Effect of weight reduction following bariatric surgery on serum visfatin and adiponectin levels in morbidly obese subjects. Obes Facts. 2013;6(2):193-202.

2. Garrido-Sánchez L, García-Fuentes E, Fernández-García D, et al. Zinc-alpha 2-glycoprotein gene expression in adipose tissue is related with insulin resistance and lipolytic genes in morbidly obese patients. PLoS One. 2012;7(3):e33264.

3. Quercioli A, Montecucco F, Pataky Z, et al. Improvement in coronary circulatory function in morbidly obese individuals after gastric bypassinduced weight loss: relation to alterations in endocannabinoids and adipocytokines. Eur Heart J. 2013;34(27):2063-73.

4. Wolf RM, Steele KE, Peterson LA, et al. Lower circulating C1q/ TNF-related protein-3 (CTRP3) levels are associated with obesity: a cross-sectional study. PLoS One. 2015;10(7):e0133955. 